STRUCTURAL BIOLOGY COMMUNICATIONS

ISSN 2053-230X

\section{Structure of the lutein-binding domain of human StARD3 at $1.74 \AA$ resolution and model of a complex with lutein}

\author{
Martin P. Horvath, ${ }^{\text {** Evan W. George, }}$, Quang T. Tran, ${ }^{a}$ Kody Baumgardner, ${ }^{\text {a }}$ \\ Gabe Zharov, ${ }^{a}$ Sarah Lee, ${ }^{a}$ Hassan Sharifzadeh,, Saeed Shihab, ${ }^{b}$ Ty Mattinson, \\ Binxing Li ${ }^{b}$ and Paul S. Bernstein ${ }^{\mathbf{b}} *$
}

Received 25 May 2016

Accepted 1 July 2016

Edited by W. N. Hunter, University of Dundee, Scotland

Keywords: carotenoid-binding protein; START domain; StARD3; lutein; protein tunnels and cavities.

PDB reference: cholesterol and lutein-binding domain of human STARD3, 5i9j

Supporting information: this article has supporting information at journals.iucr.org/f

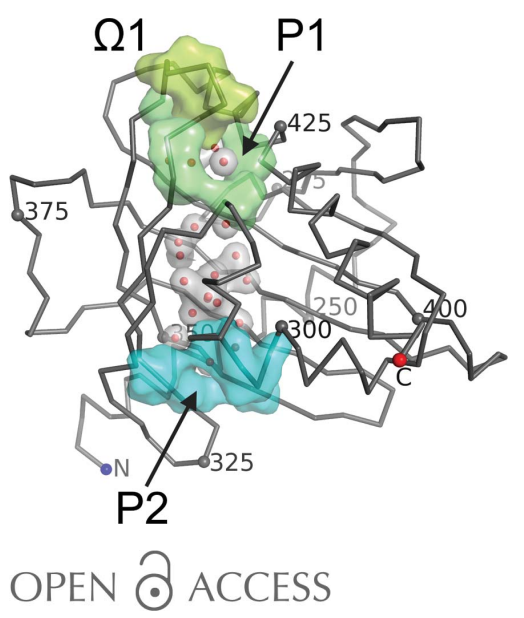

${ }^{\mathbf{a}}$ Department of Biology, University of Utah, 257 S 1400 E, Salt Lake City, UT 84112, USA, and ${ }^{\mathbf{b}}$ Department of Ophthalmology and Visual Sciences, Moran Eye Center, University of Utah School of Medicine, Salt Lake City, UT 84132, USA. *Correspondence e-mail: martin.horvath@utah.edu, paul.bernstein@hsc.utah.edu

A crystal structure of the lutein-binding domain of human StARD3 (StARrelated lipid-transfer protein 3; also known as MLN64) has been refined to $1.74 \AA$ resolution. A previous structure of the same protein determined to $2.2 \AA$ resolution highlighted homology with StARD1 and shared cholesterol-binding character. StARD3 has since been recognized as a carotenoid-binding protein in the primate retina, where its biochemical function of binding lutein with specificity appears to be well suited to recruit this photoprotective molecule. The current and previous structures correspond closely to each other (r.m.s.d. of $0.25 \AA$ ), especially in terms of the helix-grip fold constructed around a solventfilled cavity. Regions of interest were defined with alternate conformations in the current higher-resolution structure, including Arg351 found within the cavity and $\Omega 1$, a loop of four residues found just outside the cavity entrance. Models of the complex with lutein generated by rigid-body docking indicate that one of the ionone rings must protrude outside the cavity, and this insight has implications for molecular interactions with transport proteins and enzymes that act on lutein. Interestingly, models with the $\varepsilon$-ionone ring characteristic of lutein pointing towards the bottom of the cavity were associated with fewer steric clashes, suggesting that steric complementarity and ligand asymmetry may play a meso-zeaxanthin, which only have $\beta$-ionone rings.

\section{Introduction}

The macula lutea (yellow spot) at the center of the primate retina is enriched in the xanthophyll carotenoids lutein, zeaxanthin and meso-zeaxanthin. These carotenoids are thought to protect the human eye from photo-oxidative stress (Bernstein et al., 2016; Li et al., 2010; Beatty et al., 1999). Indeed, epidemiological studies and prospective clinical trials have shown that dietary intake and supplementation with lutein and zeaxanthin increase the likelihood of avoiding age-related macular degeneration (AMD), a leading cause of blindness (Seddon et al., 1994; Age-Related Eye Disease Study 2 Research Group, 2014; Wu et al., 2015; Bernstein et al., 2016). The majority of these carotenoid molecules are specifically localized in the outer plexiform layers (also known as the Henle fiber layer) of the human fovea. GSTP1 and StARD3 (also known as MLN64) have been identified as the zeaxanthin-binding and lutein-binding protein in the human macula, respectively, and these are thought to be responsible for the specific tissue distribution and stability of the ocular carotenoids (Bhosale et al., 2004; Li et al., 2011). StARD3 was initially identified as a lutein-binding protein because of its role in discriminating lutein from the other ocular carotenoids zeaxanthin and 
high degree of homology to the carotenoid-binding protein found in silkworm (Li et al., 2011). Its retina-related role was confirmed through the examination of tissue-specific expression patterns, and binding studies monitored by surface plasmon resonance (SPR) demonstrated that StARD3 binds lutein with affinity and specificity ( $\mathrm{Li}$ et al., 2011). The luteinbinding function of StARD3 resides within the C-terminal START domain comprising residues 216-444, hereafter referred to as StARD3 ${ }_{\mathrm{LBD}}$ (Li et al., 2011).

Interest in StARD3 predates the discovery of its luteinbinding function and retina-protective role. Its X-ray crystal structure was determined by the Hurley laboratory as a surrogate for StARD1 (Tsujishita \& Hurley, 2000). StARD1 mobilizes cholesterol as the first committed step in steroid biogenesis, and homology between StARD3 and StARD1 suggested that StARD3 also binds cholesterol (Watari et al., 1997; Tsujishita \& Hurley, 2000). The most remarkable structural feature characteristic of StARD3 and StARD1 is a large $\left(\sim 1000 \AA^{3}\right)$ solvent-filled cavity (Tsujishita \& Hurley, 2000; Thorsell et al., 2011).

The structural basis by which StARD3 and GSTP1 recognize, bind and protect lutein, zeaxanthin and meso-zeaxanthin is not known. With the overarching goal of providing a molecular picture for carotenoid recruitment to the human retina, we have targeted carotenoid complexes of StARD $3_{\text {LBD }}$ and GSTP1 for structure determination. Structures of these proteins in their carotenoid-complexed forms have been elusive because conditions which solubilize the hydrophobic lutein and zeaxanthin molecules require either detergent or organic solvents, and these agents have been determined to prevent crystal growth and to damage preformed crystals.

While these challenges are being resolved, we have obtained high-resolution X-ray diffraction data for the unliganded proteins and report here the structure of StARD3 $3_{\text {LBD }}$ refined to $1.74 \AA$ resolution. This structure corresponds closely to the original $2.2 \AA$ resolution structure (Tsujishita \& Hurley, 2000). The new structure includes additional solvent molecules and alternate conformations for several residues, including $\operatorname{Arg} 351$, which is located within the cavity, and residues Gly336-Gly339, which are part of the omega loop found just outside the cavity entrance. Modelbuilding experiments indicate that at least one of the ionone rings of lutein must protrude through a cavity entrance. The current structure and its lutein-complexed model are discussed in relation to mechanisms for carotenoid binding specificity and the molecular interactions necessary for the transport, protection and metabolism of carotenoids in the human eye.

\section{Methods}

2.1. Cloning of an expression system for tagless StARD3 $3_{\mathrm{LBD}}$

The tagless StARD3 $3_{\text {LBD }}$-pET-22b(+) expression system was obtained by ligation-independent cloning (Aslanidis \& de Jong, 1990; Li \& Elledge, 2007). Briefly, DNA encoding residues 216-444 of human StARD3 was amplified by PCR using a His 6 -tagged StARD3 expression plasmid as the template. Primers for this PCR reaction were designed to avoid the $\mathrm{His}_{6}$ tag and to include $\sim 20$ bp of DNA flanking the NdeI/EcoRI insertion site present in pET-22b(+). This StARD3-encoding DNA was assembled with the pET-22b(+) vector by mixing with two DNA fragments obtained by amplifying the following regions from $\mathrm{pET}-22 \mathrm{~b}(+)$ ): 287-3268 (contains an NdeI site and the lacI gene) and 3245-197 (contains an EcoRI site and the bla gene). Each of the three PCR products was purified by electrophoresis in a $0.85 \%$ agarose gel prior to mixing and transformation directly into heat-shock competent DH5 $\alpha$ Escherichia coli cells. Ampicillin-resistant colonies were screened by restriction-enzyme digestion and DNA sequencing of miniprep DNA. The resulting T7 promoter-driven expression vector encodes residues 216-444 of StARD3, as confirmed by mass spectrometry.

\subsection{Purification of tagless StARD $3_{L B D}$}

The lutein-binding domain of StARD3 was expressed in E. coli Origami B (DE3) cells (Novagen) and purified by ammonium sulfate fractionation followed by ion-exchange and size-exclusion chromatography. The concentration of StARD $3_{\text {LBD }}$ was determined from the absorbance at $280 \mathrm{~nm}$ using an extinction coefficient of $1.1 \mathrm{ml} \mathrm{mg}^{-1} \mathrm{~cm}^{-1}$ $\left(0.028 \mu M^{-1} \mathrm{~cm}^{-1}\right)$ calculated by the method of Gill \& von Hippel (1989).

2.2.1. Growth of bacterial cultures. Bacterial cultures were cultivated with shaking in $2 \times \mathrm{YT}$ medium supplemented with $30 \mathrm{~m} M$ potassium phosphate $\mathrm{pH} 7.5,5 \mathrm{~m} M$ glucose, $10 \mu \mathrm{g} \mathrm{ml}^{-1}$ kanamycin, $12.5 \mu \mathrm{g} \mathrm{ml}^{-1}$ tetracycline and $500 \mu \mathrm{g} \mathrm{ml}^{-1}$ ampicillin. A $750 \mathrm{ml}$ culture was expanded at $310 \mathrm{~K}$. At an $\mathrm{OD}_{600 \mathrm{~nm}}$ of $0.7-0.9$, the culture was chilled on ice before adding IPTG to a final concentration of $100 \mu \mathrm{M}$. Induction proceeded at $290 \mathrm{~K}$ with shaking for $24-30 \mathrm{~h}$ prior to harvesting the cells by centrifugation at $4000 \mathrm{~g}$. The cells were washed in $10 \mathrm{~m} M$ Tris, $1 \mathrm{~m} M$ EDTA $\mathrm{pH} 8.2,0.5 \mathrm{~m} M$ phenylmethylsulfonyl fluoride (PMSF) and collected by centrifugation prior to freezing at $253 \mathrm{~K}$.

2.2.2. Ammonium sulfate precipitation. Frozen cells were submerged in $100 \mathrm{~g}$ lysis buffer consisting of $0.1 \mathrm{M}$ sodium chloride, $0.05 M$ Tris pH 8, $1 \mathrm{~m} M$ EDTA. Upon thawing, cells were lysed by sonication on ice. The lysate was centrifuged at $20000 \mathrm{~g}$, and the supernatant was collected in an ice-cold glass beaker. Solid ammonium sulfate was added slowly with stirring on ice to achieve $30 \%$ saturation (17.5 g ammonium sulfate per $100 \mathrm{~g}$ supernatant). After $30 \mathrm{~min}$, the sample was centrifuged at $15000 \mathrm{~g}$ for $20 \mathrm{~min}$. Additional ammonium sulfate was added slowly with stirring on ice to achieve $65 \%$ saturation (an additional $22.5 \mathrm{~g}$ ammonium sulfate per $100 \mathrm{~g}$ supernatant). After $40 \mathrm{~min}$, the second ammonium sulfate fraction was collected by centrifugation at $15000 \mathrm{~g}$ for $15 \mathrm{~min}$. The pellet was dissolved by adding $20 \mathrm{ml}$ of freshly prepared, ice-cold dialysis buffer consisting of $50 \mathrm{~m} M$ sodium chloride, $25 \mathrm{~m} M$ HEPES pH 6.5, $0.25 \mathrm{~m} M$ EDTA, $1 \mathrm{~m} M$ dithiothreitol (DTT). The sample was dialyzed against 1.51 dialysis buffer at $277 \mathrm{~K}$ without stirring overnight. 
2.2.3. Ion-exchange and size-exclusion chromatography. The dialysate was centrifuged at $20000 \mathrm{~g}$ in an ice-cold rotor for $20 \mathrm{~min}$, filtered through a low-protein-binding $0.45 \mu \mathrm{m}$ filter and loaded by gravity onto two $2 \mathrm{ml}$ SP Sepharose columns in parallel, each equilibrated in dialysis buffer. The average flow rate was approximately $1 \mathrm{ml} \mathrm{min}^{-1}$. Columns were washed with $10 \mathrm{ml}$ dialysis buffer and eluted by increasing the concentration of sodium chloride stepwise with $5 \mathrm{ml}$ volume per step. Fractions enriched in StARD3 ${ }_{\text {LBD }}$ were identified by SDS-PAGE stained with Coomassie, pooled and concentrated to a volume of $0.3 \mathrm{ml}$ before filtering using a $0.45 \mu \mathrm{m}$ Nanosep spin filter (Pall Life Sciences) and injecting onto a size-exclusion column equilibrated with SEC buffer consisting of $20 \mathrm{~m} M$ Tris $\mathrm{pH} 7.5,150 \mathrm{~m} M$ sodium chloride, $2 \mathrm{~m} M$ DTT. Fractions enriched in StARD3 ${ }_{\mathrm{LBD}}$ protein were pooled and concentrated to $8 \mathrm{mg} \mathrm{ml}^{-1}$ (Fig. 1a).

\subsection{Crystallography}

2.3.1. Crystallization. Crystals of StARD $3_{\text {LBD }}$ were obtained by the hanging-drop vapor-diffusion method as described previously (Tsujishita \& Hurley, 2000). Well solutions consisting of $0.1 M$ CHES $\mathrm{pH}$ 8.6-9.4, 0.2 $M$ lithium sulfate, $0.8-0.96 M$ sodium/potassium tartrate, $0.01 M$ DTT were combined with equal volumes $(1.5-3 \mu \mathrm{l})$ of protein solution. Rod-shaped crystals appeared within $2 \mathrm{~d}$ and grew

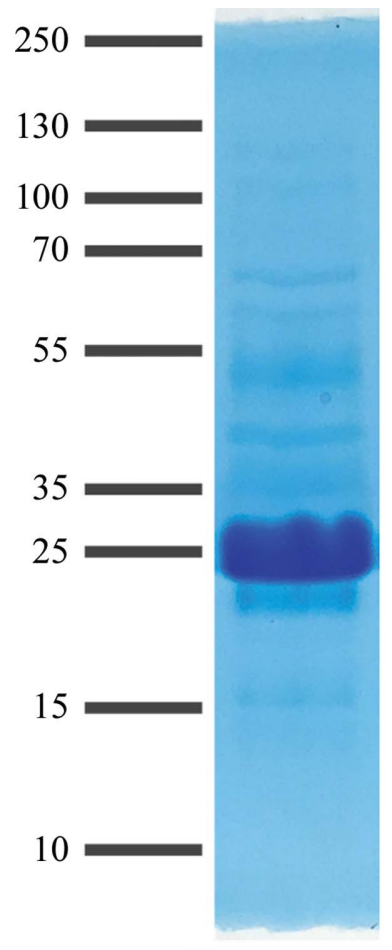

(a)
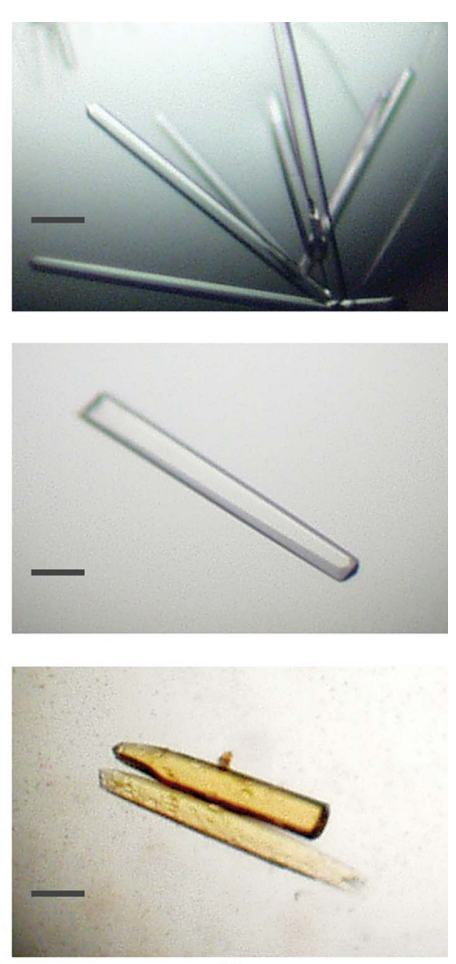

(b)
Figure 1

Purity and crystals of StARD3 $3_{\text {LBD }}$ (a) Purified StARD3 $3_{\text {LBD }}(15 \mu \mathrm{g})$ was analyzed by SDS-PAGE and stained with Coomassie Blue. The most prominent $(\sim 90 \%$ ) protein co-elutes with a $25 \mathrm{kDa}$ molecular-weight marker (expected mass $29953 \mathrm{Da}$ ). (b) Rod-shaped crystals grew in 1$2 \mathrm{~d}$. The scale bar indicates $100 \mu \mathrm{m}$. Crystals of StARD3 ${ }_{\mathrm{LBD}}$ are colorless (top, middle) and acquire a golden color when stored in solutions also containing powdered lutein (bottom).
Table 1

Data collection and processing.

Values in parentheses are for the outer shell.
Diffraction source

Wavelength $(\AA)$

Temperature (K)

Detector

Exposure time per image (s)

Space group

$a, b, c(\AA)$

$\alpha, \beta, \gamma\left({ }^{\circ}\right)$

Mosaicity $\left({ }^{\circ}\right)$

Resolution range $(\AA)$

Total No. of reflections

No. of unique reflections

Completeness (\%)

Multiplicity

$\langle I / \sigma(I)\rangle$

$R_{\text {r.i.m. }}$

Overall $B$ factor from Wilson plot $\left(\AA^{2}\right)$
Rotation range per image $\left(^{\circ}\right)$
Beamline 12.3.1, ALS

1.11583

100

ADSC Quantum 315r

0.5

0.2 and 1.5

$P 3_{1} 21$

$83.39,83.39,82.19$

$90,90,120$

$0.14-0.21$

41.7-1.74 (1.79-1.74)

301752 (16789)

$34160(2466)$

99.5 (98.6)

$8.8(6.8)$

$25.8(2.4)$

$0.048(0.879)$

32.6 to a maximum size within one week at room temperature (Fig. 1b). Crystals were moved to matched solutions additionally containing $15-20 \%$ ethylene glycol prior to cooling in liquid propane and storage under liquid nitrogen.

2.3.2. Data collection. Crystals were measured in oscillation mode on the SIBYLS beamline 12.3.1 at the Advanced Light Source (Classen et al., 2013) using synchrotron radiation tuned to $1.116 \AA$. The rod morphology made it possible to reposition the crystal periodically during the course of data collection so as to obtain complete and redundant data without overexposing any one portion. Indexing and strategy development was accomplished with $H K L$-2000 (Otwinowski \& Minor, 1997). Complete data sets were processed with $X D S$ and XSCALE (Kabsch, 2010a,b).

2.3.3. Refinement. Phases were obtained by placing PDB entry 1em2 (Tsujishita \& Hurley, 2000) into the nearly isomorphous unit cell by rigid-body refinement. Refinement continued with several iterative cycles of positional and temperature-factor adjustment with PHENIX and model adjustment with Coot (Adams et al., 2010; Emsley et al., 2010). Early refinement rounds additionally applied torsion-angle simulated annealing with a temperature protocol descending from $1500 \mathrm{~K}$ to a final temperature of $50 \mathrm{~K}$.

\subsection{Model construction of the complex with lutein}

Structural models of protein-complexed lutein were obtained from the light-harvesting complexes of spinach and pea with the following PDB entries: 1rwt (Liu et al., 2004), 3p19 (Pan et al., 2011), 4xk8 (Qin et al., 2015), 4y28 (Mazor et al., 2015) and 3jcu (Wei et al., 2016). Selected lutein molecules were manually positioned within the StARD3 structure to generate a number of docked templates. An ensemble of 27324 structures was constructed by superposition of the 40 experimentally determined lutein structures from PDB entries 1rwt, 3pl9, 4xk8 and 3jcu onto these docked template molecules with variations of least-squares alignments either directly or with inverting molecule orientation. Ensemble members were scored by determining the number of clashes 
and the number of potential hydrogen bonds with use of a $\mathrm{C}$ language program written by MPH. Frequency analysis to detect the orientation preference was limited to the 8469 ensemble members that belonged to the 'one portal' set and that scored at least one potential hydrogen bond.

\section{Results}

\subsection{Protein expression and crystallization}

Initial attempts to prepare crystals of StARD $3_{\text {LBD }}$, purified as described previously (Tsujishita \& Hurley, 2000), yielded small crystals with poor diffraction quality. We therefore cloned a T7 promoter-driven expression system that produces StARD3 $3_{\text {LBD }}$ without a tag, with the idea that the challenge in producing crystals may have related to the removal of the $\mathrm{His}_{6}$ tag by protease treatment. Purification by means of ammonium sulfate fractionation and two chromatography steps (and no proteolysis treatment) yielded $5 \mathrm{mg}$ protein from 0.751 bacterial culture (Fig. 1a). The yield and purity were lower than that obtained with the tagged version; however, the resulting protein readily produced large $(50 \times 50 \times 500 \mu \mathrm{m})$ rod-shaped crystals (Fig. 1b) which diffracted synchrotron radiation to $1.7 \AA$ resolution (Table 1 ).

\subsection{Structure of StARD3 $3_{L B D}$}

3.2.1. Structure determination. The structure of StARD3 $3_{\text {LBD }}$ was determined by refinement against the newly measured $1.74 \AA$ resolution data, starting with rigid-body placement of a previously determined structure (Tsujishita \& Hurley, 2000). The starting model (PDB entry 1em2) is $99 \%$ identical to the wild-type StARD3 $3_{\mathrm{LBD}}$ determined in the current work, and both proteins crystallized in the same $P 3_{1} 21$ space group with highly comparable unit-cell parameters. The higher resolution limit reported here probably reflects differences in crystal size (the present crystals are twice as large in each dimension), differences in synchrotron source flux (ALS beamline 12.3.1 for the present study versus NSLS beamline $\mathrm{X} 4 \mathrm{~A}$ for the previous study) and improvements in detector capabilities (ADSC Q315r versus ADSC Q4). The final structural model includes residues 231-444 of StARD3 (the coordinates for residues 216-230 were omitted because no

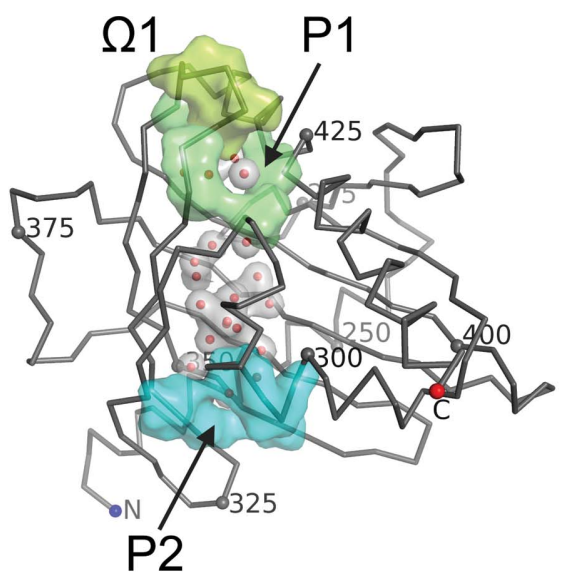

(a)

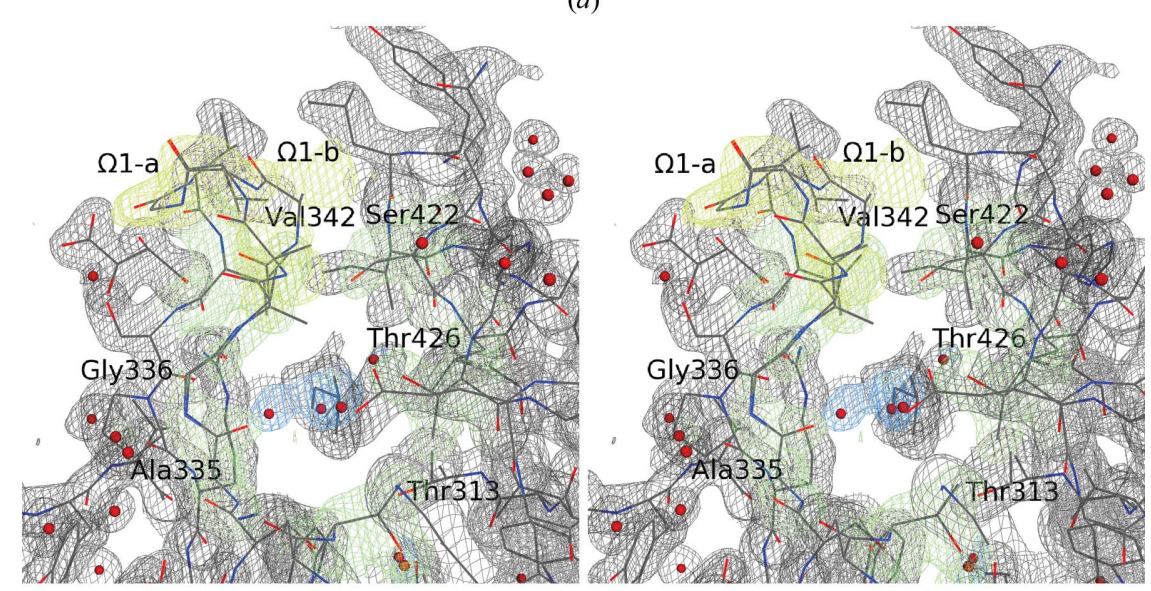

(b)

\section{Figure 2}

Structure overview and representative electron density for StARD $3_{\mathrm{LBD}}(a)$ The $\mathrm{C}^{\alpha}$ trace of StARD3 is shown along with surfaces for key structural features: the omega loop $(\Omega 1$, lemon), portal 1 (P1, lime), portal $2(\mathrm{P} 2$, cyan) and solvent molecules located inside the cavity (red spheres inside gray molecular surfaces). (b) A stereoview of electron density and protein structure at portal 1 is shown. Electron density was calculated as a simulatedannealing composite OMIT map with $2\left|F_{\mathrm{o}}\right|-\left|F_{\mathrm{c}}\right|$ coefficients and is contoured at 1.1 $\sigma$. Map color indicates residues belonging to the omega loop (lemon), portal 1 (lime) and tunnel solvent (blue). Both conformations of the omega loop are shown ( $\Omega 1$-a and $\Omega 1$-b). Residues lining portal 1 are labeled (Thr313, Ala335, Gly336, Val342, Ser422 and Thr426). 
electron density was observed), 224 water molecules, three molecules of ethylene glycol, one sulfate ion and one molecule of L-(+)-tartaric acid. The $R_{\text {work }}$ and $R_{\text {free }}$ values are 0.169 and 0.192 , respectively. Table 2 reports statistical measures for model validation.

3.2.2. Structure overview. Fig. 2 shows an overview of the protein as well as representative electron density. As described previously (Tsujishita \& Hurley, 2000), StARD $3_{\text {LBD }}$ adopts the helix-grip fold with a nine-stranded curved $\beta$-sheet and three $\alpha$-helices coalescing around a large solvent-filled cavity. In helix-grip proteins the cavity entrance is guarded by an omega loop ( $\Omega 1$ ) that connects two $\beta$-strands ( $\beta 5$ and $\beta 6$ in StARD $3_{\text {LBD }}$ ). The current structure is highly correlated with the $2.2 \AA$ resolution structure (Tsujishita \& Hurley, 2000). Superposition yields a root-mean-square deviation (r.m.s.d.) of $0.25 \AA$ for $210 \mathrm{C}^{\alpha}$ atoms. The main differences in the structure involve the restoration of the wild-type residues Met307, Phe388 and Met427 (these residues were substituted with selenomethionine in PDB entry 1em2) and the modeling of 17 residues with alternate conformations, including Arg351, which is inside the cavity, and residues Gly336-Gly339, which are part of $\Omega 1$ near the cavity entrance.

3.2.3. Tunnel-like cavity. A striking feature of StARD $3_{\text {LBD }}$ is the prominent tunnel-like cavity lined by hydrophobic and polar residues with sufficient volume to accommodate a molecule of cholesterol, as demonstrated by modeling (Tsujishita \& Hurley, 2000). The tunnel-like cavity measures $\sim 20 \AA$ long from end to end, curves slightly and accommodates 18 solvent molecules, one of which is an ethylene glycol in the current structure (Fig. 2). Electron-density maps did not reveal any larger molecules located inside the cavity. The cavity communicates with bulk solvent through two openings, which we will call portal 1 and portal 2 (Fig. 2). Portal 1 is wider in diameter compared with portal $2(4 \times 8 \AA$ for portal 1 versus $2 \times 4 \AA$ for portal 2 ; distances are measured between solvent-accessible surfaces). Indeed, structural descriptions of helix-grip proteins generally speak of only one cavity entrance.
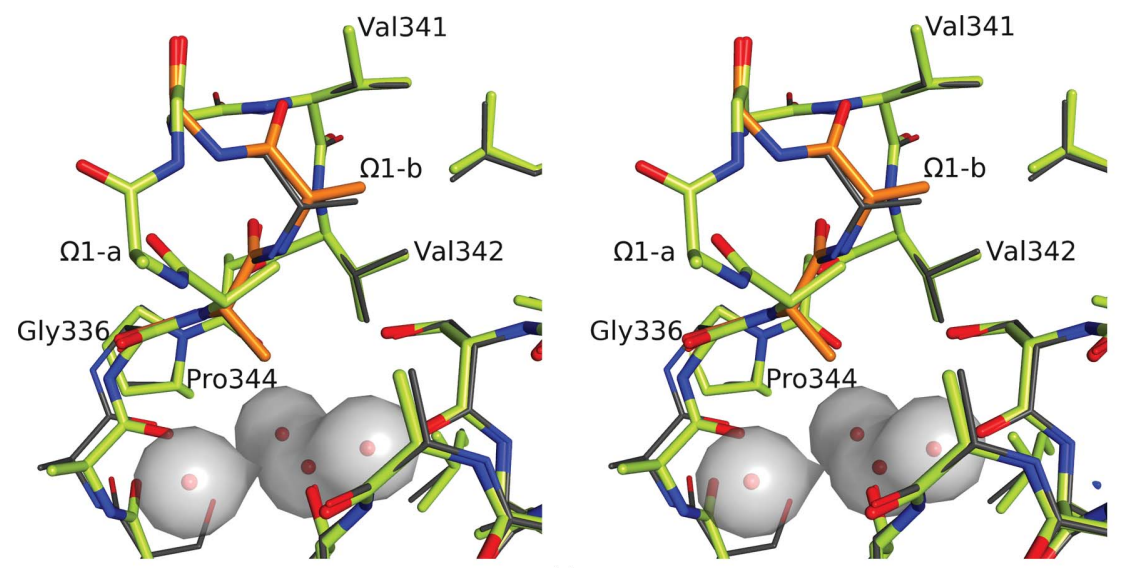

(a)

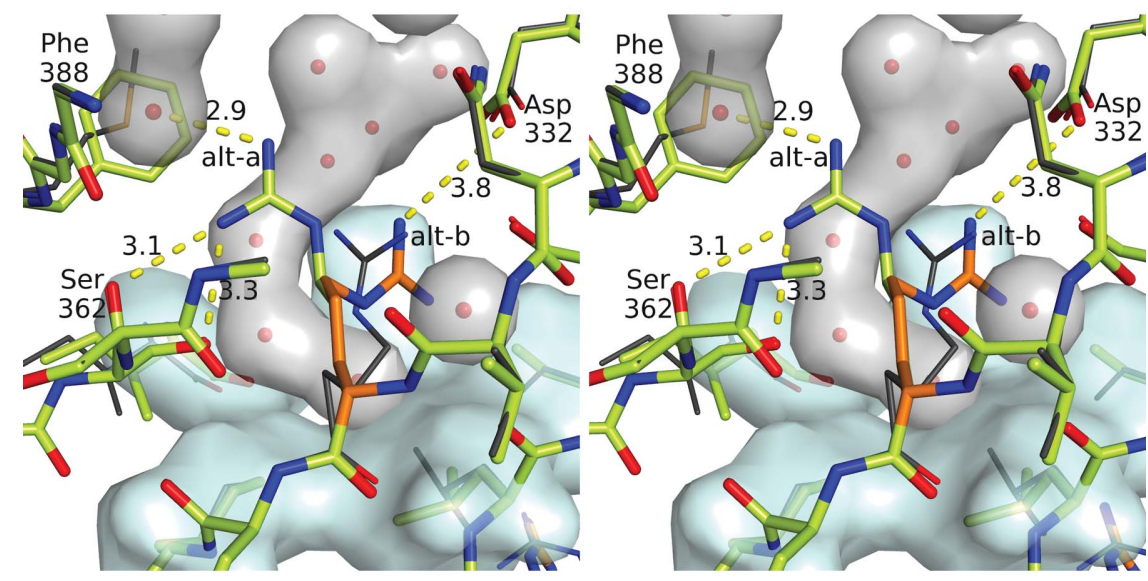

(b)

\section{Figure 3}

Alternate conformations for the omega loop $(a)$ and $\operatorname{Arg} 351(b)$ as stereoviews. The structure of StARD $3_{\text {LBD }}$ reported here is shown superimposed with the previously determined structure (PDB entry 1em2; charcoal $\mathrm{C}$ atoms). Protein residues, including the A conformation, are shown with $\mathrm{C}$ atoms colored lime. The $\mathrm{B}$ conformation is shown with $\mathrm{C}$ atoms colored orange. Solvent molecules located in the tunnel-like cavity are shown as red spheres inside gray molecular surfaces. (a) Ala338 experiences the largest displacement ( $6.6 \AA$ for $\mathrm{C}^{\beta}$ ), moving away from the cavity entrance upon switching from the $\Omega 1$-b to the $\Omega 1$-a conformation. (b) Hydrogen bonds and salt bridges evident for Arg351 in each of two alternate conformations are indicated with dashed yellow lines and distance measurements $(\AA)$. In this panel, portal 2, shown as a molecular surface, comprises residues Pro304, Ile319, Leu328 and Ile353, as well as the main-chain atoms of residues 301-303. Residue 388 is Phe (wild type) in the current structure and selenomethionine in the previously reported structure. For clarity, some residues and solvent molecules are not shown. 
3.2.4. Alternate conformations. Structural flexibility is evident for residues located near the cavity portals. A segment of four residues (Gly336, Ala337, Ala338 and Gly339) was associated with weaker-than-average electron density along two alternative chain paths, suggesting that the $\Omega 1$ loop is capable of movement just outside portal 1 . These residues were modeled in two alternative conformations (Fig. $3 a$ ), one of which was seen in the original structure $(\Omega 1-b$; occupancy $=$ $0.44)$. The other conformation $(\Omega 1$-a; occupancy $=0.56)$ differs principally by a flip in the peptide bond connecting Ala338 and Gly339, which results in a maximal $6.6 \AA$ displacement away from the tunnel opening experienced by $\mathrm{C}^{\beta}$ of residue Ala338 (Figs. $2 b$ and $3 a$ ). Smaller displacements are observed for the neighboring residue Ala337, which rotates by approximately $90^{\circ}$. In addition to the alternate conformations observed outside portal 1, the side chain of Arg351 was resolved as two alternative conformations inside the tunnel close to portal 2 (Fig. $3 b$ ). One of these reaches across the cavity to form a salt bridge with Asp332 and is most similar to the conformation of this residue reported previously (Arg351-b; occupancy $=0.43$ ). The second conformation (Arg351-a; occupancy $=0.57$ ) adopts a different rotamer and forms hydrogen bonds to solvent molecules and the carbonyl and hydroxyl $\mathrm{O}$ atoms of Ser362. Structural flexibility in these regions may be necessary for the binding of bulky ligands such as lutein. Alternate conformations are also evident for residues 276-280, which are located on the surface of StARD3 $3_{\text {LBD }}$ and do not directly impact tunnel accessibility.

\subsection{Building a model of the complex with lutein}

3.3.1. Lutein structure. Lutein $\left(\mathrm{C}_{40} \mathrm{H}_{56} \mathrm{O}_{2}\right)$ belongs to the xanthophyll carotenoids synthesized by plants and bacteria.<smiles>CC(C=CC=C(C)C=CC1=C(C)C(P)[C@@H](O)CC1(C)C)=CC=CC=C(C)C=CC=C(C)C=C[C@@]1(C)C[C@@H](O)[C@H](O)C=C1C</smiles>

(a)<smiles>CC1=C(/C=C/C(C)=C/C=C/C(C)=C/C=C/C=C(C)/C=C/C=C(C)/C=C/C2=C(C)C(=P)[C@H](O)CC2(C)C)C(C)(C)C[C@@H](O)C1</smiles>

(b)<smiles>CC1=C(/C=C/C(C)=C/C=C/C(C)=C/C=C/C=C(C)/C=C/C=C(C)/C=C/C2=C(C)C(=P)[C@@H](O)CC2(C)C)C(C)(C)C[C@@H](O)C1</smiles>

(c)

Figure 4

Chemical structures of lutein $(a)$, zeaxanthin $(b)$ and meso-zeaxanthin $(c)$. The ionone rings of lutein have different shapes. The $\beta$-ring contains a single stereocenter $(3 R)$; the $\varepsilon$-ring contains two stereocenters $\left(3^{\prime} R, 6^{\prime} R\right)$. Asymmetry in the ionone shape distinguishes lutein from the other ocular xanthophylls and may contribute to selectivity for lutein and exclusion of zeaxanthin and meso-zeaxanthin, which each have two $\beta$-ionone rings ( $3 R$ and $3^{\prime} R$ in zeaxanthin; $3 R$ and $3^{\prime} S$ in meso-zeaxanthin).
Table 2

Structure solution and refinement.

Values in parentheses are for the outer shell.

\begin{tabular}{ll}
\hline Resolution range $(\AA)$ & $37.2-1.74(1.79-1.74)$ \\
Completeness $(\%)$ & $99.5(98.6)$ \\
$\sigma$ Cutoff & -3 \\
No. of reflections, working set & $32447(2624)$ \\
No. of reflections, test set & $1712(141)$ \\
Final $R_{\text {cryst }}$ & $0.170(0.255)$ \\
Final $R_{\text {free }}$ & $0.192(0.320)$ \\
Coordinate error $\dagger(\AA)$ & 0.18 \\
No. of non-H atoms & \\
$\quad$ Protein & 1799 \\
$\quad$ Ligand and ions & 27 \\
$\quad$ Water & 224 \\
$\quad$ Total & 2050 \\
R.m.s. deviations & \\
$\quad$ Bonds $(\AA)$ & 0.013 \\
$\quad$ Angles $\left({ }^{\circ}\right)$ & 1.16 \\
Average $B$ factors $\left(\AA^{2}\right)$ & \\
$\quad$ Protein & 29.5 \\
$\quad$ Ligand and ions & 43.3 \\
$\quad$ Water & 36.7 \\
Ramachandran plot & \\
$\quad$ Most favored $(\%)$ & 97 \\
Allowed $(\%)$ & 3 \\
MolProbity score $\ddagger$ & 1.4 \\
Clashscore & 4.4
\end{tabular}

$\dagger$ Maximum-likelihood-based. See equation (19) in Lunin et al. (2002). $\ddagger$ Chen et al. (2010).

Two ionone rings, each bearing a hydroxyl group, are connected by a long polyene backbone. The molecular structure includes three defined stereocenters (Fig. 4). We examined the structures of protein-complexed lutein molecules found as part of the light-harvesting complexes from spinach and pea (Liu et al., 2004; Pan et al., 2011; Qin et al., 2015; Wei et al., 2016). All examples show curvature in the polyene backbone, steric complementarity with surfaces of the proteins, especially at ionone ring-contacting pockets, and two or three hydrogen bonds formed between each of the hydroxyl groups of lutein and acceptor and donor groups presented by the chlorophyll-binding protein subunits. The average length measured between hydroxyl $\mathrm{O}$ atoms is $30.5 \pm 0.2 \AA$. The size and shape of lutein are thus closely matched to the size and shape of the cavity found in StARD $3_{\mathrm{LBD}}$, but it would be impossible to completely fit the entire molecule into the cavity. Interestingly, crevices and protrusions of the protein found just outside each portal appear to be articulated to specifically accommodate some type of ligand. We propose that structural features close to and outside one or both of the portals make contact with an ionone ring, similar to the ionone ring-binding pockets observed in light-harvesting complex structures (Liu et al., 2004; Pan et al., 2011; Qin et al., 2015; Mazor et al., 2015; Wei et al., 2016).

3.3.2. Rigid-body docking. To test this idea, lutein molecules were docked within the StARD3 ${ }_{\text {LBD }}$ structure (Fig. 5). Several different lutein molecules were tested, each derived from an experimental structure of a light-harvesting complex. The omega loop was assumed to be in its $\Omega 1$-a conformation, and Arg351 was likewise restricted to the conformation that 
hydrogen bonds to Ser362 (not salt-bridged with Asp332) because these conformations appeared to be most accommodating with respect to avoiding steric clashes. Except for translation and rotation, no structural adjustments were permitted in either the lutein ligand or the StARD3 $3_{\mathrm{LBD}}$ protein. Each docking outcome was scored for clashes (unfavorable, closer than $3 \AA$ contact) and hydrogen bonds (favorable, 2.0-4.2 $\AA$ distance from hydroxyl O atom to donor or acceptor). We realise that these distance cutoffs are generous by comparison with strict stereochemical rules, but judge them to be reasonable when considering that adjustments in structure are to be anticipated. The 'winner' incurred fewer steric clashes than any of the other outcomes and realised two hydrogen bonds, one for each of the two hydroxyl groups of lutein (Fig. 5).

3.3.3. Orientation preference. The ensemble of 27324 docked lutein molecules included four overlapping sets: a set that penetrated both portals ('two portals'; $n=14256$ ), a set that penetrated only the larger portal 1 ('one portal'; $n=13068$ ), a set oriented so that the $\varepsilon$-ionone ring with two
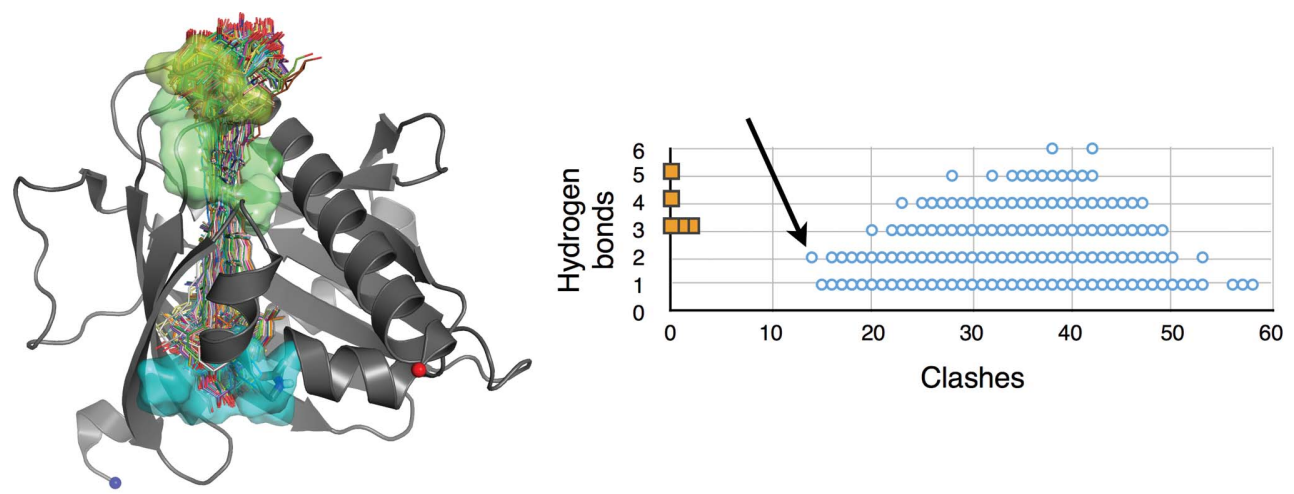

(a)
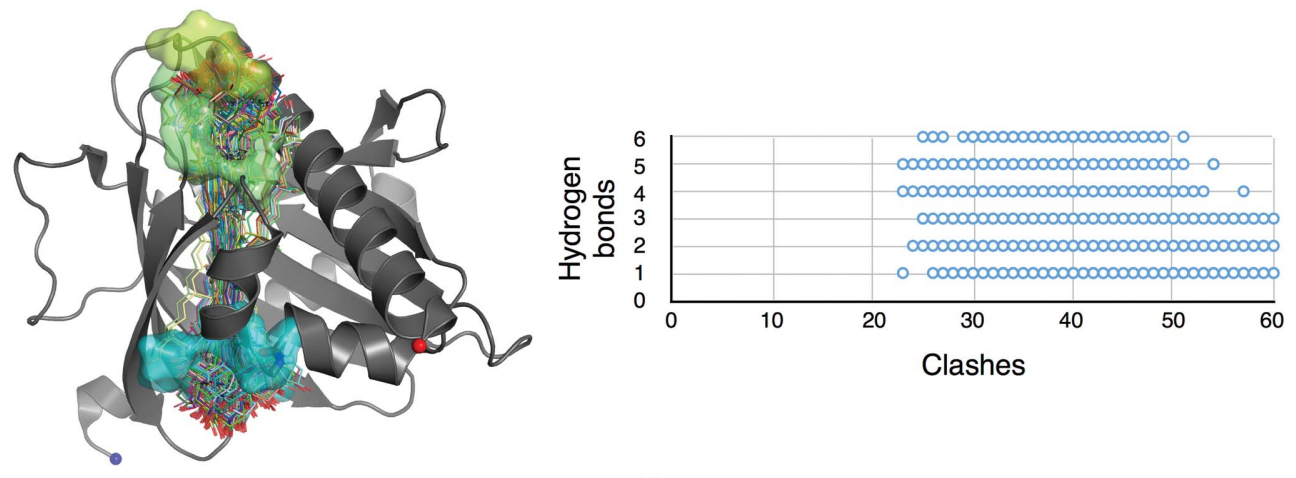

(b)

Figure 5

Lutein molecules docked with StARD3. The ensemble can be divided into two sets shown in $(a)$ and $(b)$. (a) Members of this set protrude only through portal 1 ( $n=13$ 068; 'one portal'). (b) Members of the other set breach both portals ( $n=14256$; 'two portals'). Clashes and potential hydrogen bonds are plotted for members of each set that scored at least one but not more than six hydrogen bonds (blue open circles). For reference, scores measured for experimentally determined structures of protein-complexed lutein molecules are also plotted [gold-filled squares in $(a)$ ]. The best scoring molecules from the 'two portals' set incurred 23 clashes. The overall 'winner' belonged to the 'one portal' set shown in $(a)$, incurred 14 clashes and realised two potential hydrogen bonds (an arrow points to the data point characterizing this winner).

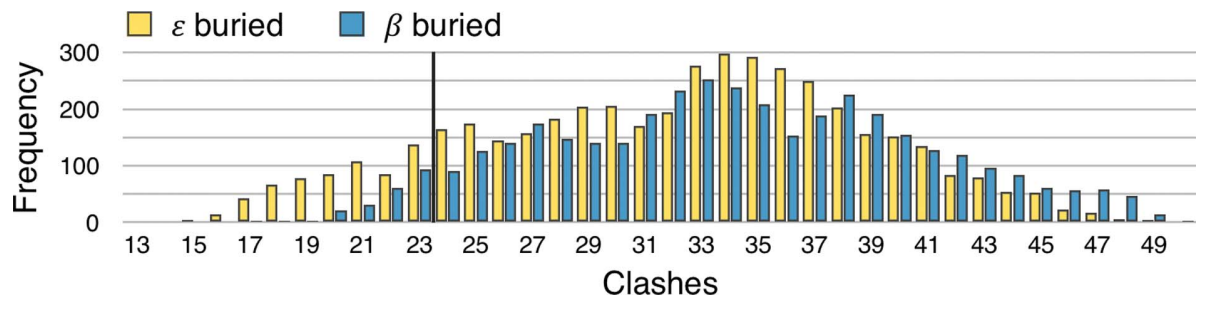

Figure 6

Orientation preference for lutein. The frequency of observing docked lutein-StARD $3_{\mathrm{LBD}}$ complexes is plotted as a function of steric clashes incurred for members of the 'one portal' set with at least one potential hydrogen bond. Within the top $10 \%$ scoring group (fewer than 23 clashes; 836 of 8469 outcomes), the frequency for lutein molecules oriented with the $\varepsilon$-ionone ring buried deep inside the cavity (yellow bars; $n=620$ ) was 2.9 -fold higher compared with the frequency for the opposite orientation with the $\beta$-ionone ring buried (blue bars; $n=216$ ). This frequency difference meets the criteria for statistical significance with $p<0.00001$ for a chi-squared test. Similarly strong frequency differences indicating that it is easier to place the $\varepsilon$-ionone ring inside the tunnel-like cavity and the $\beta$-ionone ring outside portal 1 were observed if the analysis was repeated with $\Omega 1$ and Arg351 adopting their alternate conformations or with $\Omega 1$ deleted and Arg351 truncated to an alanine residue. 
chiral centers was closest to portal $1(n=13662)$ and a set oriented in the opposite direction with this $\varepsilon$ ring close to portal $2(n=13662)$. The distribution of clashscores indicates a strong tendency for high-scoring models to belong to the 'one portal' set (Fig. 5) and oriented so that the $\varepsilon$ ring is buried close to portal 2 (Fig. 6). Indeed, the absolute winner of the docking experiment belonged to both of these two sets and was seen with its $\beta$-ionone ring extending past portal 1 to nestle into a binding surface defined by the $\Omega 1$ loop with apparent steric and hydrogen-bonding complementarity (Fig. 7). To test the source of the observed orientation preference, we repeated the analysis with residues belonging to $\Omega 1$ and Arg351 in their alternate conformations and found that the number of clashes increased (on average +3 for $\Omega 1-b$ and +1 for Arg 351-b), yet the strong $(p<0.00001)$ orientation preference was retained. Deleting residues belonging to $\Omega 1$ and truncating $\operatorname{Arg} 351$ to alanine shifted the frequency distributions to fewer clashes $(-8$ on deleting $\Omega 1 ;-3$ on truncating Arg351) and preserved the approximately threefold orientation preference favoring burial of the $\varepsilon$ ring close to portal 2.

\section{Discussion}

\subsection{Co-crystallization of carotenoid-binding proteins}

A molecular understanding of how photoprotective carotenoids are recruited to the human retina will potentially help with the prevention and treatment of AMD. The carotenoidbinding proteins GSTP1 and StARD3 bind zeaxanthin and lutein, respectively, with affinity and specificity (Bhosale et al., 2004; Li et al., 2011). Efforts to co-crystallize the xanthophyllprotein complexes are under way; however, it has been difficult to find conditions under which the hydrophobic ligands are soluble and also compatible with the crystalline protein state. The most promising route to an experimentally determined co-crystal structure may emerge by combining powdered lutein and preformed crystals of $S t A R D 3_{\text {LBD }}$ in aqueous solutions containing trace amounts of detergent. The crystals appear to acquire a golden-brown color over several weeks (Fig. 1b) yet retain X-ray diffraction performance.

\subsection{Structure of StARD3 $3_{L B D}$ and model of its complex with lutein}

While these soaking experiments were in progress, we measured X-ray diffraction for crystals of apo StARD $3_{\mathrm{LBD}}$ and generated models of the lutein-StARD3 complex by rigid-body docking. StARD3 crystals diffracted X-rays to relatively high resolution limits at a synchrotron source, allowing the detection of alternative conformations for a residue of interest found inside the central cavity of this helixgrip folded protein and for the $\Omega 1$ loop close to the entrance to the cavity. The winner of the rigid-body docking analysis incurs 14 steric overlaps with atoms of the protein and potentially makes two hydrogen bonds. By comparison, experimentally determined structures of lutein in complex with chlorophyll-binding proteins experience at most two steric clashes and make between three and five hydrogen bonds. The docking procedure applied here should be viewed as a coarse-grid search and caution is warranted in making detailed inferences from the modeling outcome.

4.2.1. A portion of lutein must extend beyond the tunnellike cavity. The lutein-docking experiment is nevertheless illuminating in several respects. Comparison of the molecular size of lutein with that of the cavity leads to the inescapable conclusion that at least a portion of the lutein molecule must bind outside the cavity. Indeed, surfaces just outside the cavity appear to be complementary to the shape and hydrogenbonding capacity of its $\beta$-ionone ring (Fig. 7). This predicted mode of binding is unprecedented for START proteins and other helix-grip folded proteins. In all structures where a ligand complex is available, the ligand is completely ensconced within the central cavity (Thorsell et al., 2011; Létourneau et

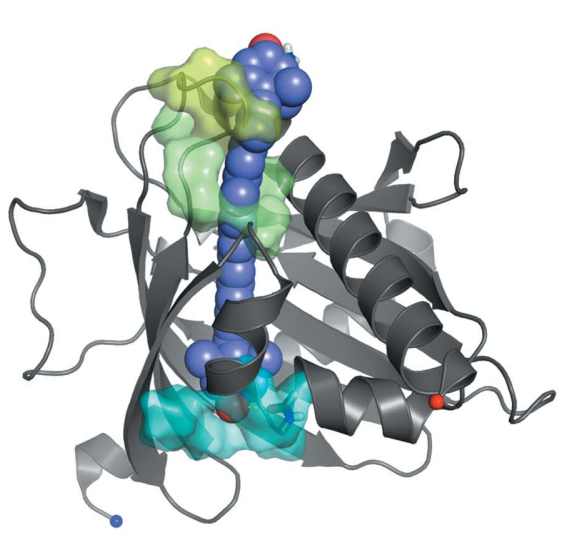

(a)

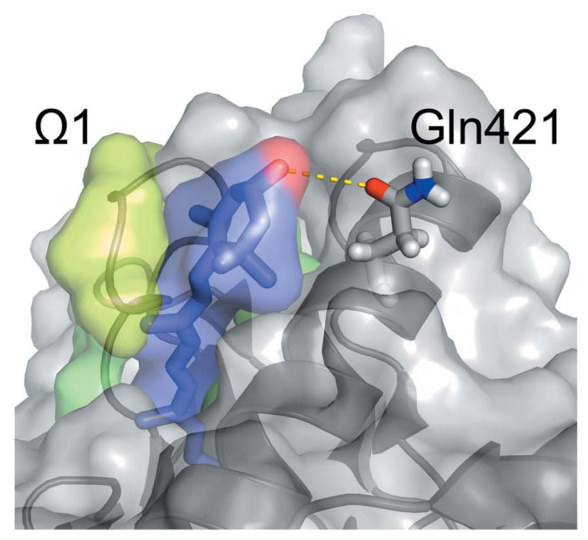

(b)

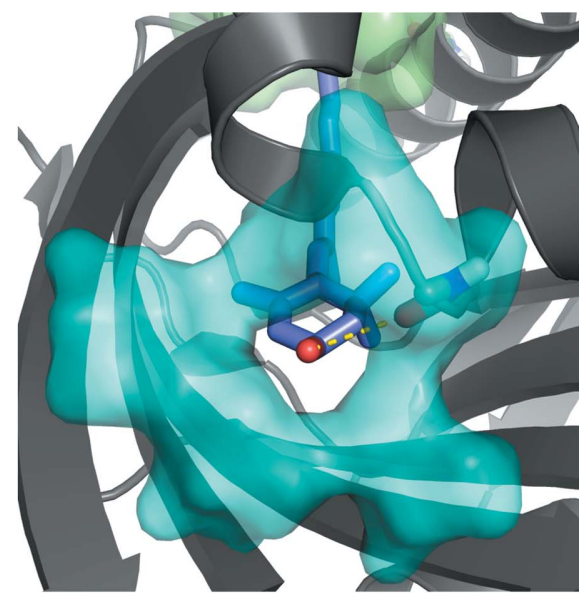

(c)

Figure 7

'Winner' lutein molecule docked with StARD3. (a) Overview of the StARD3 ${ }_{\text {LBD }}$ structure shows the best-scoring lutein candidate from the 'one portal' set. (b) Steric complementarity and a potential hydrogen bond to Gln421 are apparent outside portal 1 for the overall 'winner' lutein molecule. (c) The 'winner' lutein molecule positions the hydroxyl group of the $\varepsilon$-ionone ring just inside portal 2, where it finds a potential hydrogen-bonding partner with the peptide bond connecting residues Ile301 and Leu302. 
al., 2015). Leaving a portion of the molecule on the outside of the protein as proposed here has important implications for the molecular interactions necessary for the transport and metabolism of lutein.

4.2.2. Orientation preference and implications for ligand recognition. An unexpected outcome of the docking experiment was that the $\varepsilon$-ionone ring, with two chiral centers, appears to fit better inside the cavity, with fewer clashes and additional potential hydrogen bonds associated with this orientation, on average, in the ensemble of docking trials (see Fig. 6). This pattern suggests an interesting model for ligand selectivity: the binding-pocket asymmetry apparent for the lutein-StARD3 model may positively identify lutein, which is inherently asymmetrical with $\beta$-ionone and $\varepsilon$-ionone rings, and exclude the closely related xanthophylls zeaxanthin and meso-zeaxanthin, which exhibit symmetry and have two $\beta$-ionone rings. Consistent with this idea that symmetry plays a role in xanthophyll selectivity, GSTP1, which is homodimeric, is the zeaxanthin- and meso-zeaxanthin-binding protein in the human retina (Bhosale et al., 2004). The winner of the luteinStARD3 docking experiment makes several close contacts with the $\Omega 1$ loop (Fig. 7) and the side chain of Arg351, suggesting that these contribute to the molecular-recognition properties of StARD3. A strong orientation preference was retained in the docking experiment with $\Omega 1$ and the side chain of Arg351 adopting their alternate conformations or even when they were removed. These results lead us to believe that molecular interactions involving surfaces throughout and outside the tunnel-like cavity, including $\Omega 1$ and Arg351, probably act cooperatively to select a particular binding orientation and positively identify lutein.

4.2.3. Second portal of StARD3. Although two portals potentially allow solvent to access the cavity at opposite ends of StARD3, one of these is much larger. The minimum number of clashes encountered for lutein molecules docked so as to penetrate both entrances was 23 , which is nine more than that observed for the best-scoring model docked so as to penetrate just the larger portal. We therefore predict that lutein binds deep within the cavity, that one of the ionone rings protrudes through the larger opening which is guarded by the $\Omega 1$ loop, and that there is a close approach to but no breach of the smaller opening. It will be interesting to compare these predictions with an experimental structure of the co-complex and/or more refined modeling experiments that apply energyrelaxation methods (Das \& Baker, 2008; Leaver-Fay et al., 2011).

4.2.4. Allosteric trigger point. StARD $3_{\mathrm{LBD}}$ belongs to the helix-grip protein family, and protein members with structural homology include START proteins and allergen proteins, as well as several biosynthetic enzymes. Inspection of these structures reveals that StARD3 and StARD1 differ from the others in sharing identical or highly conserved residues at three positions that appear to be important for ligand binding: Arg at position 351 (numbered as in StARD3; 188 in StARD1), an acidic residue at position 332 (Asp in StARD3; Glu169 in StARD1) and Gln at position 421 (position 258 in StARD1). In other START-family members these three resi- dues are variable, and all three are not found together. The lutein-docking results suggest that Gln421 may be positioned so as to make a hydrogen bond to a hydroxyl group of lutein outside portal 1 (Fig. 7). Electron-density maps, now calculated to $1.74 \AA$ resolution, show that Arg351 adopts two alternative conformations (Fig. $3 b$ ), one of which forms a salt bridge to Asp332. Interestingly, this salt-bridged conformation is probably incompatible with lutein occupancy owing to steric overlap. Based on these observations, we suggest that the salt bridge found in cavities of StARD3 and StARD1 may act as an allosteric trigger point to communicate ligand binding to other components of the steroid-generating apparatus in the case of StARD1, and to retinal proteins and enzymes involved with xanthophyll transport and metabolism in the case of StARD3.

\section{Conclusion}

The structure of the lutein-binding domain of StARD3 has been determined to $1.74 \AA$ resolution, revealing alternative conformations for elements of protein structure that appear to be critical for ligand binding. Modeling experiments indicate that the biologically relevant ligand must protrude from the cavity entrance because it is not possible to completely fit a $30 \AA$ long molecule of lutein into a cavity that measures only $20 \AA$. Asymmetry in the tunnel-like cavity may play a role in selecting lutein with its $\beta$ - and $\varepsilon$-ionone rings and discriminating against the other ocular carotenoids, zeaxanthin and meso-zeaxanthin, which each have two $\beta$-ionone rings.

\section{Acknowledgements}

We thank Dr Scott Classen and Jane Tanamachi for assistance with data collection at the SIBYLS beamline. Dr James Hurley generously supplied the original expression system for StARD3 ${ }_{\text {LBD }}$. This work was supported by the National Institutes of Health (NIH; EY11600 to PSB and EY14600 to the Department of Ophthalmology). EWG was supported by a UROP fellowship through the Office of Undergraduate Research at the University of Utah. SL was supported by a Bioscience Summer Research Program with the Department of Biology, University of Utah. The SIBYLS beamline at the Advanced Light Source, Lawrence Berkeley National Laboratory is supported in part by the US Department of Energy (DOE) program Integrated Diffraction Analysis Technologies (IDAT) and the DOE program Molecular Assemblies Genes and Genomics Integrated Efficiently (MAGGIE) under Contract No. DE-AC02- 05CH11231. The Moran Eye Center has received an unrestricted departmental grant from Research to Prevent Blindness.

\section{References}

Adams, P. D. et al. (2010). Acta Cryst. D66, 213-221.

Age-Related Eye Disease Study 2 Research Group (2014). JAMA Ophthalmol. 132, 142-149.

Aslanidis, C. \& de Jong, P. J. (1990). Nucleic Acids Res. 18, 6069-6074.

Beatty, S., Boulton, M., Henson, D., Koh, H. H. \& Murray, I. J. (1999). Br. J. Ophthalmol. 83, 867-877. 
Bernstein, P. S., Li, B., Vachali, P. P., Gorusupudi, A., Shyam, R., Henriksen, B. S. \& Nolan, J. M. (2016). Prog. Retin. Eye Res. 50, 34-66.

Bhosale, P., Larson, A. J., Frederick, J. M., Southwick, K., Thulin, C. D. \& Bernstein, P. S. (2004). J. Biol. Chem. 279, 49447-49454.

Chen, V. B., Arendall, W. B., Headd, J. J., Keedy, D. A., Immormino, R. M., Kapral, G. J., Murray, L. W., Richardson, J. S. \& Richardson, D. C. (2010). Acta Cryst. D66, 12-21.

Classen, S., Hura, G. L., Holton, J. M., Rambo, R. P., Rodic, I., McGuire, P. J., Dyer, K., Hammel, M., Meigs, G., Frankel, K. A. \& Tainer, J. A. (2013). J. Appl. Cryst. 46, 1-13.

Das, R. \& Baker, D. (2008). Annu. Rev. Biochem. 77, 363-382.

Emsley, P., Lohkamp, B., Scott, W. G. \& Cowtan, K. (2010). Acta Cryst. D66, 486-501.

Gill, S. C. \& von Hippel, P. H. (1989). Anal. Biochem. 182, 319-326.

Kabsch, W. (2010a). Acta Cryst. D66, 125-132.

Kabsch, W. (2010b). Acta Cryst. D66, 133-144.

Leaver-Fay, A. et al. (2011). Methods Enzymol. 487, 545-574.

Létourneau, D., Lefebvre, A., Lavigne, P. \& LeHoux, J.-G. (2015). Mol. Cell. Endocrinol. 408, 53-61.

Li, B., Ahmed, F. \& Bernstein, P. S. (2010). Arch. Biochem. Biophys. 504, 56-60.

Li, M. Z. \& Elledge, S. J. (2007). Nature Methods, 4, 251-256.

Li, B., Vachali, P., Frederick, J. M. \& Bernstein, P. S. (2011). Biochemistry, 50, 2541-2549.
Liu, Z., Yan, H., Wang, K., Kuang, T., Zhang, J., Gui, L., An, X. \& Chang, W. (2004). Nature (London), 428, 287-292.

Lunin, V. Y., Afonine, P. V. \& Urzhumtsev, A. G. (2002). Acta Cryst. A58, 270-282.

Mazor, Y., Borovikova, A. \& Nelson, N. (2015). Elife, 4, e07433.

Otwinowski, Z. \& Minor, W. (1997). Methods Enzymol. 276, 307-326.

Pan, X., Li, M., Wan, T., Wang, L., Jia, C., Hou, Z., Zhao, X., Zhang, J. \& Chang, W. (2011). Nature Struct. Mol. Biol. 18, 309-315.

Qin, X., Suga, M., Kuang, T. \& Shen, J. R. (2015). Science, 348, 989-995.

Seddon, J. M., Ajani, U. A., Sperduto, R. D., Hiller, R., Blair, N., Burton, T. C., Farber, M. D., Gragoudas, E. S., Haller, J., Miller, D. T., Yannuzzi, L. A. \& Willett, W. (1994). JAMA, 272, 1413-1420.

Thorsell, A.-G., Lee, W. H., Persson, C., Siponen, M. I., Nilsson, M., Busam, R. D., Kotenyova, T., Schüler, H. \& Lehtiö, L. (2011). PLoS One, 6, e19521.

Tsujishita, Y. \& Hurley, J. H. (2000). Nature Struct. Biol. 7, 408414.

Watari, H., Arakane, F., Moog-Lutz, C., Kallen, C. B., Tomasetto, C., Gerton, G. L., Rio, M. C., Baker, M. E. \& Strauss, J. F. (1997). Proc. Natl Acad. Sci. USA, 94, 8462-8467.

Wei, X., Su, X., Cao, P., Liu, X., Chang, W., Li, M., Zhang, X. \& Liu, Z. (2016). Nature (London), 534, 69-74.

Wu, J., Cho, E., Willett, W. C., Sastry, S. M. \& Schaumberg, D. A. (2015). JAMA Ophthalmol. 133, 1415-1424. 